\title{
Editorial
}

\section{Discrete and Dynamic Optimization Problems in Operation Management}

\author{
Xiang Li, ${ }^{1}$ Chen Zhou, ${ }^{2}$ and Xiaochen Sun ${ }^{3}$ \\ ${ }^{1}$ College of Economic and Social Development, Nankai University, Tianjin 300071, China \\ ${ }^{2}$ School of Industrial and Systems Engineering, Georgia Institute of Technology, 755 Ferst Drive, NW Atlanta, GA 30332-0205, USA \\ ${ }^{3}$ Department of Mathematics, School of Science, Tianjin University, Tianjin 300072, China \\ Correspondence should be addressed to Xiang Li; xiangli@nankai.edu.cn
}

Received 27 March 2013; Accepted 27 March 2013

Copyright (C) 2013 Xiang Li et al. This is an open access article distributed under the Creative Commons Attribution License, which permits unrestricted use, distribution, and reproduction in any medium, provided the original work is properly cited.

\section{Introduction}

Discrete and dynamic optimization is a significant methodology that has been widely applied to operation management fields. This issue on discrete and dynamic optimization problems in operation management aims at an all-around research and the state-of-the-art theoretical, numerical, and practical achievements that contribute to this field. This issue contains 21 papers, with the following features.

\section{On Financial Engineering}

C. Zhang and X. Rong study the optimal investment strategies of defined contribution pension, with the stochastic interest rate and stochastic salary. $H$. Zhao et al. consider the optimal investment problem for an insurer in a complete market. Optimal strategies are obtained via martingale approach, and computational results can provide practical advice to insurers. Y. Li and G. Liu shed light on the dynamic proportional reinsurance in a two-dimensional compound Poissons' risk model.

\section{On Production and Distribution Optimization}

Y. Zhou and J. Sun investigate an inventory replenishment problem with component substitution and product substitution simultaneously in the product-updated system. X. Sun et al. study an inventory replenishment and production planning problem for a two-period inventory system with dependent returns and demands. L. Liao et al. develop mathematical models of inventory-distribution routing problem for two-echelon agriculture products distribution network. J. Ma and G. Sun use a mutation Ant Colony Algorithm to solve the milk-run VRP with fastest completion time. W. Xue et al. study the joint inventory and sales-effort management problems of a retailer in a broad context and investigate the optimal policies for a single-item, periodic-review system.

\section{On Supply Chain Optimization}

$\mathrm{L} . \mathrm{Xu}$ et al. introduce the reference effect into the seacargo supply chain and study a multiple-period contract problem between the carrier and forwarder. G. Sun develops a dynamic model in a one-supplier-one-retailer fresh agricultural products supply chain that experiences supply disruptions during the planning horizon. W. Hu and J. $\mathrm{Li}$ integrate a retailer's return policy and a supplier's buy-back policy within a supply chain with return logistics modeling framework. F. Hu et al. analyze a coordination problem with random demands and random supplies with disruptions in a supply chain system with one retailer and two suppliers. W. Liu et al. built a time scheduling model of logistics service supply chain, which is constrained by the service order time requirement. Y. Luo et al. study a process of product renewal in a supply chain with one manufacturer and one retailer, with optimal product pricing strategies derived. J. Wei et al. study the pricing decisions of a two-echelon supply chain with one 
manufacturer and duopolistic retailers in fuzzy environment. J. Zhao et al. develop the optimal pricing and remanufacturing decisions problem of a fuzzy closed-loop supply chain.

\section{On Scheduling and Queuing Systems}

X. Li and J. Li consider the workload process of the M/G/1 queuing system. Under different cases, they analyze the explicit criteria of the geometric rate of convergence and the geometric decay of stationary tail. R. Zhang considers a parallel machine scheduling problem with random processing/setup times and adjustable production rates. S. Lv and J. Li shed light on the multiserver repairable queuing system with variable breakdown rates, which widely exists in practical life.

\section{Others}

Z. Liao et al. study the innovation diffusion problem with the affection of urbanization, propose a dynamical innovation diffusion model with fuzzy coefficient, and use the shifting rate of people from rural areas stepping into urban areas to show the process of urbanization. L. Fu et al. use universal combinatorial operation model to describe the logic relationship and gave a flexible logic control method, which is useful to realize an effective control for complex system.

Xiang $\mathrm{Li}$

Chen Zhou

Xiaochen Sun 


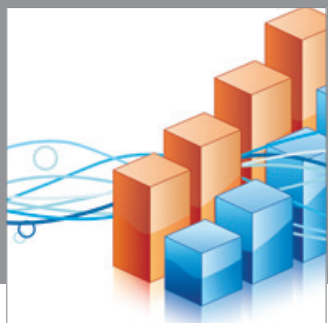

Advances in

Operations Research

mansans

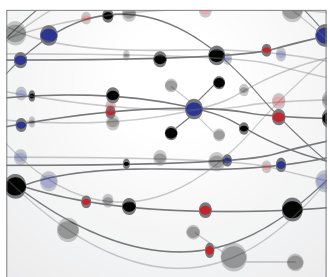

The Scientific World Journal
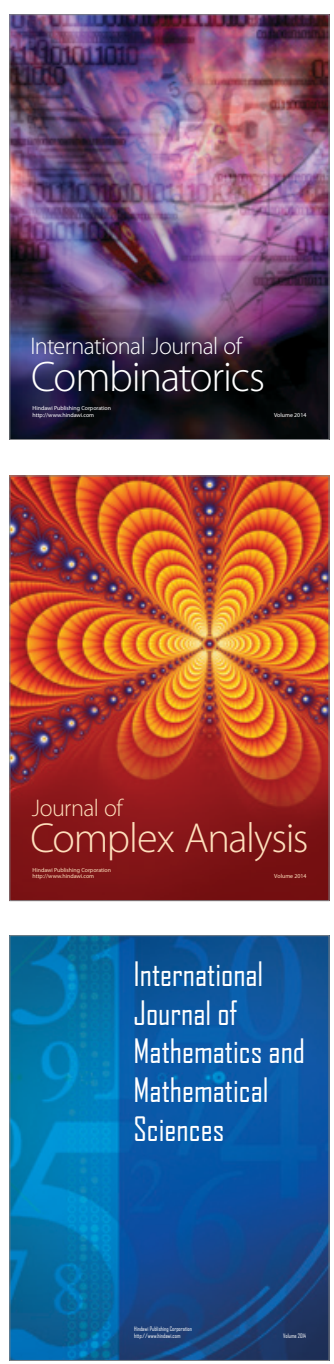
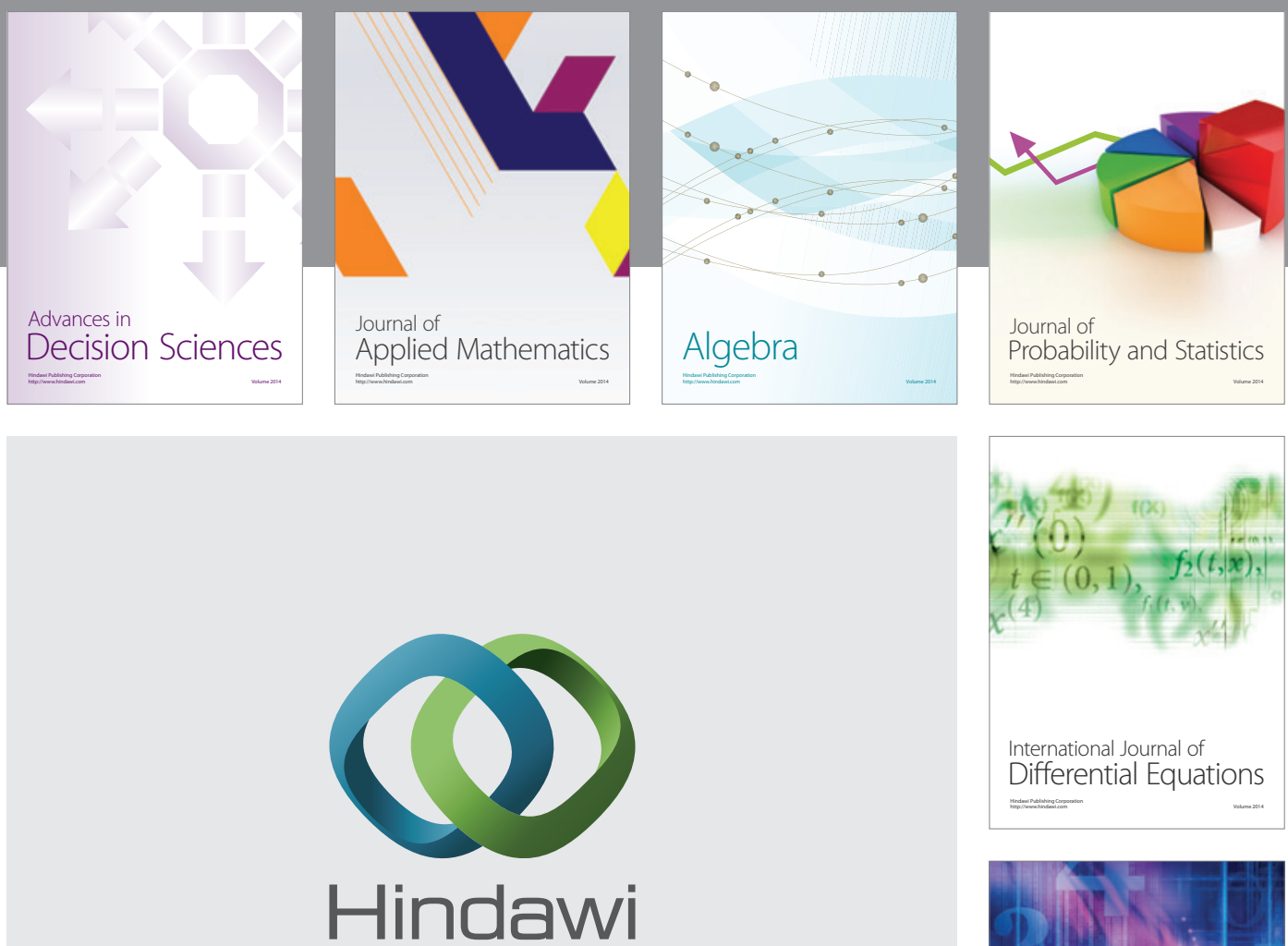

Submit your manuscripts at http://www.hindawi.com
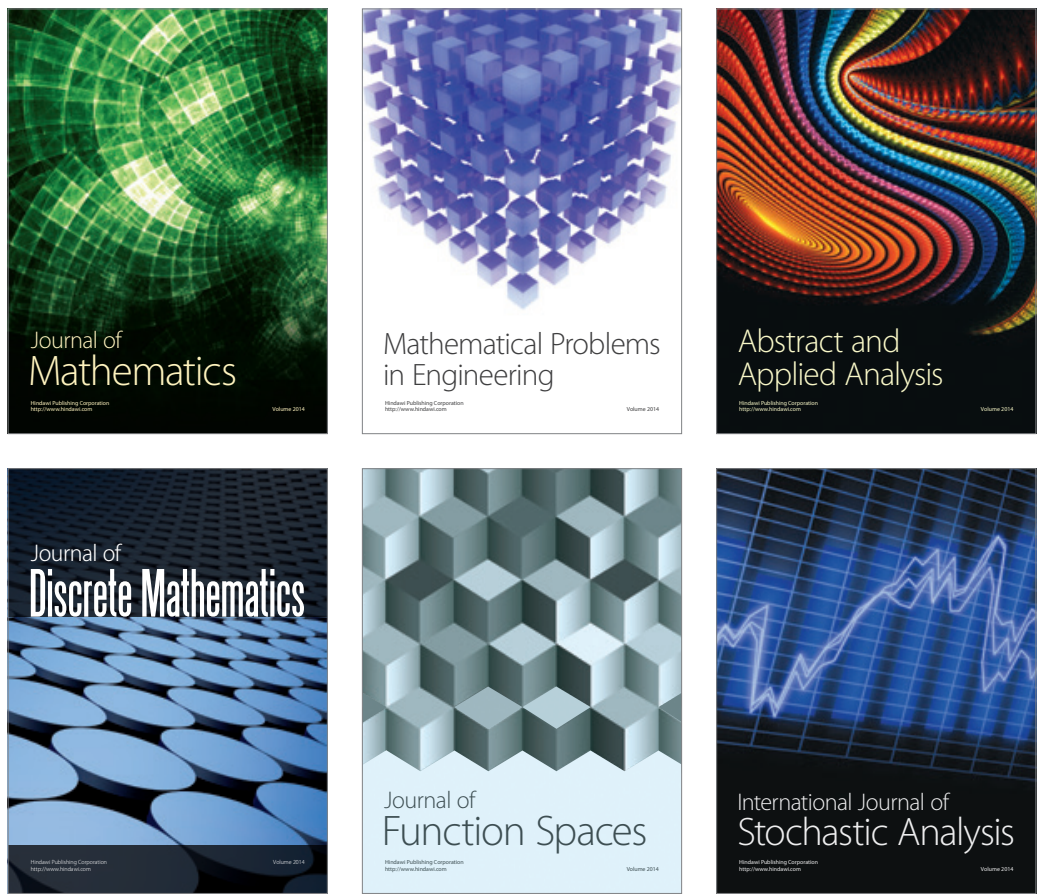

Journal of

Function Spaces

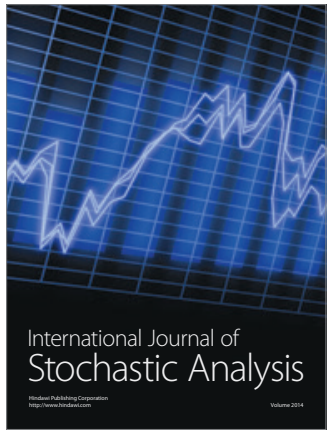

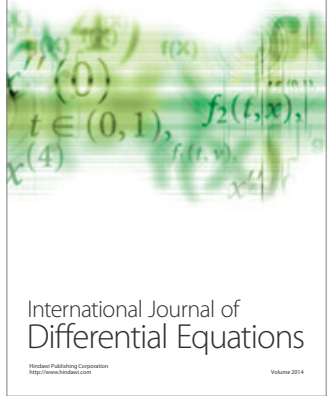
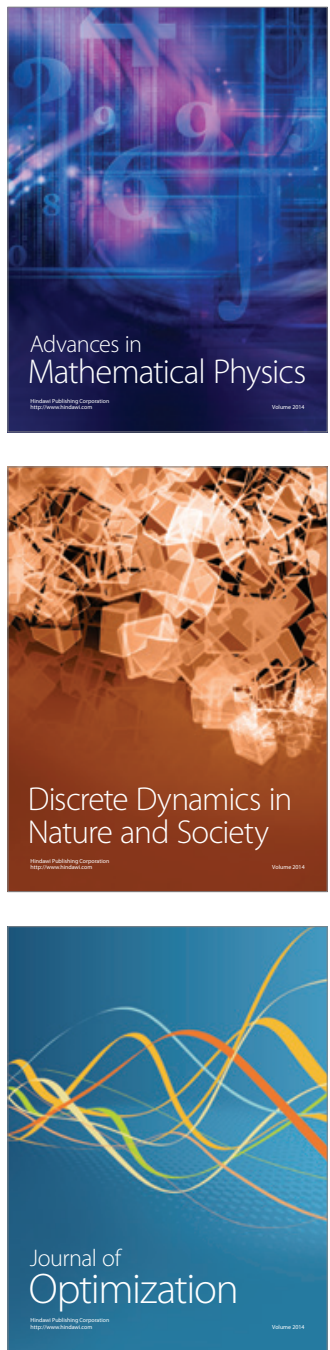